\title{
The $v$ process in the innermost supernova ejecta
}

\author{
Andre Sieverding ${ }^{1,2, \star}$, Gabriel Martínez Pinedo ${ }^{1,2}$, Karlheinz Langanke ${ }^{2,1}$, J. Austin Harris ${ }^{3,4}$, \\ and W. Raphael Hix ${ }^{4,5}$, \\ ${ }^{1}$ Institute for Nuclear Physics (Theory Department), Darmstadt University of Technology, Schlossgarten- \\ straße 2, 64289 Darmstadt, Germany \\ ${ }^{2}$ Helmholtz Center for Heavy lon Research (GSI), Planckstr. 1, 64259 Darmstadt, Germany \\ ${ }^{3}$ Nuclear Science Division, Lawrence Berkeley National Laboratory, Berkeley, CA 94720, USA \\ ${ }^{4}$ Physics Division, Oak Ridge National Laboratory, P.O. Box 2008, Oak Ridge, TN 37831-6354, USA \\ ${ }^{5}$ Department of Physics and Astronomy, University of Tennessee, Knoxville, TN 37996-1200, USA
}

\begin{abstract}
The neutrino-induced nucleosynthesis ( $v$ process) in supernova explosions of massive stars of solar metallicity with initial main sequence masses between 13 and $30 \mathrm{M}_{\odot}$ has been studied with an analytic explosion model using a new extensive set of neutrino-nucleus cross-sections and spectral properties that agree with modern supernova simulations. The production factors for the nuclei ${ }^{7} \mathrm{Li},{ }^{11} \mathrm{~B},{ }^{19} \mathrm{~F},{ }^{138} \mathrm{La}$ and ${ }^{180} \mathrm{Ta}$, are still significantly enhanced but do not reproduce the full solar abundances. We study the possible contribution of the innermost supernova eject to the production of the light elements ${ }^{7} \mathrm{Li}$ and ${ }^{11} \mathrm{~B}$ with tracer particles based on a $2 \mathrm{D}$ supernova simulation of a 12 $\mathrm{M}_{\odot}$ progenitor and conclude, that a contribution exists but is negligible for the total yield for this explosion model.
\end{abstract}

\section{Introduction}

Core Collapse Supernovae are the most luminous source of neutrinos in nature that we know today. All flavors of neutrinos are emitted from the hot and dense environment of a collapsing massive star. Even though neutrinos interact with matter only via the charged- and neutral current channels of the weak interaction, they appear in the Supernova environment in such tremendous numbers that they not only help to revive the explosion shock wave but also interact with the material in the outer layers of star, that has been processed by the hydrostatic burning stages during the life of the progenitor star. The neutrinos coming from the deep interior have high energies compared to the thermal energies encountered in the stellar mantle and can either be captured on nuclei in an inverse $\beta$ decay or induce spallation reactions, i.e. lead to nuclear excitations that decay by particle emission. The effect of these interactions on the final composition of the ejecta is called the $v$ process and has been suggested to contribute to the production of ${ }^{7} \mathrm{Li},{ }^{11} \mathrm{~B}$ and ${ }^{19} \mathrm{~F}$ as well as ${ }^{138} \mathrm{La}$ and ${ }^{180} \mathrm{Ta}$. This process has been included in most of the recent nucleosynthesis surveys (e.g. [1]).

\footnotetext{
^e-mail: asiever@theorie.ikp.physik.tu-darmstadt.de
} 
Table 1. Production factors relative to solar abundances from reference [7], normalized to ${ }^{16} \mathrm{O}$ production averaged with a Salpeter IMF over the progenitor range 13-30 $\mathrm{M}_{\odot}$. Shown are the results obtained without neutrinos, with the set of low neutrino energies that are in agreement with recent supernova simulations and with the traditional choice of high neutrino energies as described in the text. For ${ }^{180} \mathrm{Ta}$ is assumed that $35 \%$ of its amount at $200 \mathrm{~s}$ after the onset of the explosion remain in the long-lived isomeric state [8]. For a detailed discussion of this aspect see also [9].

\begin{tabular}{lccccc} 
Nucleus & ${ }^{7} \mathrm{Li}$ & ${ }^{11} \mathrm{~B}$ & ${ }^{19} \mathrm{~F}$ & ${ }^{138} \mathrm{La}$ & ${ }^{180} \mathrm{Ta}$ \\
\hline \hline no $v$ & 0.001 & 0.01 & 0.34 & 0.13 & 0.17 \\
Low energies & 0.05 & 0.26 & 0.42 & 0.56 & 0.47 \\
High energies & 0.36 & 0.99 & 0.56 & 1.19 & 0.84 \\
\hline
\end{tabular}

\section{The $v$ process in a simple 1D model}

One of the first detailed studies of the $v$ process was presented in ref. [2] using an analytic approximation to describe the thermodynamic conditions of the supernova shock heating and expansion for a range of the compositional layers that are expected to be found in massive star as a result of the hydrostatic burning stages. Following this approach we have calculated the nucleosynthesis for a range of progenitor models with initial masses between 13 and $30 \mathrm{M}_{\odot}$ from ref. [1] with the same analytic model that is based on a radiation dominated shock and assuming a total thermal explosion energy of $10^{51} \mathrm{erg}$. The evolution of the nuclear abundances starting from the composition of the progenitor models is followed with a nuclear reaction network including 1988 species and an extensive set of neutrino-nucleus cross section partially based on experimental data for key reactions and including multi-particle emission channels. For more details see also ref. [3]. The neutrinos are assumed to follow Fermi-Dirac distributions with chemical potential $\mu_{v}=0$.

Previous studies of the $v$ process ( e.g. ref. [4]) have considered neutrinos with relatively high average energies of $\left.\left.\left\langle E_{v_{e}}\right\rangle=12.6 \mathrm{MeV}\right),\left\langle E_{\bar{v}_{e}}\right\rangle=15.8 \mathrm{MeV}\right)$, and $\left\langle E_{\nu_{\mu, \tau}}\right\rangle=18.8 \mathrm{MeV}$. This choice of neutrino energies will in the following be denoted as high energies. Recent advances in supernova simulations that include more extensive and accurate neutrino opacities within the neutrino transport in multiple dimensions have lead to the conclusion that the neutrino energies could even be much lower than these values (e.g. [5]). Therefore we consider here a set of low energies with $\left\langle E_{v_{e}}\right\rangle=$ 8.8 MeV), $\left.\left\langle E_{\bar{v}_{e}}\right\rangle=12.6 \mathrm{MeV}\right)$, and $\left\langle E_{v_{\mu, \tau}}\right\rangle=12.6 \mathrm{MeV}$. Table 1 shows the resulting production factors normalized to ${ }^{16} \mathrm{O}$ production and averaged over the range of progenitors considered here with a Salpeter-like initial mass function (IMF) following $d n_{*} / d m_{*} \propto m_{*}^{-1.35}$. With the lower energies the effect of the $v$ process is reduced compared to the case of the more energetic spectra from previous studies. However, several of this nuclei can also have contributions from other processes, e.g. ref. [6] suggests that up to $70 \%$ of the solar abundance of ${ }^{11} \mathrm{~B}$ can be provided by Cosmic Ray spallation in the interstellar medium. Hence, a production factor of around 0.3 from supernova ejecta is consistent with the solar abundance. The $v$ process cannot contribute significantly to the production of ${ }^{7} \mathrm{Li}$ when the lower neutrino energies are considered. The production of light elements in the outer regions can also be affected by neutrino flavor transformations, see e.g. [10, 11]. Supernovae of massive stars can contribute, but are probably not the primary source for the solar abundance of ${ }^{19} \mathrm{~F}$. The heavier nuclei ${ }^{138} \mathrm{La}$ and ${ }^{180} \mathrm{Ta}$ are still produced up to half of their solar abundance. A large fraction of the total yield of ${ }^{11} \mathrm{~B}$ is produced in the C-rich layer via ${ }^{12} \mathrm{C}\left(v, v^{\prime} p / n\right)$. Another big contribution comes from the reaction chain ${ }^{3} \mathrm{He}(\alpha, \gamma){ }^{7} \mathrm{Be}(\alpha, \gamma){ }^{11} \mathrm{C}$ and ${ }^{3} \mathrm{H}(\alpha, \gamma){ }^{7} \mathrm{Li}(\alpha, \gamma){ }^{11} \mathrm{~B}$ which is made possible by ${ }^{4} \mathrm{He}\left(v, v^{\prime} n / p\right)$. This process also produces ${ }^{7} \mathrm{Li}$, mostly as ${ }^{7} \mathrm{Be}$ and it occurs mostly in the lower He shell but can also in the innermost regions where the composition results from an $\alpha$ rich freeze out from NSE in Si burning. Large parts of this material could eventually fall back and get accreted onto the neutron star. This effect is commonly accounted for in 1D calculation by assuming an artificial 

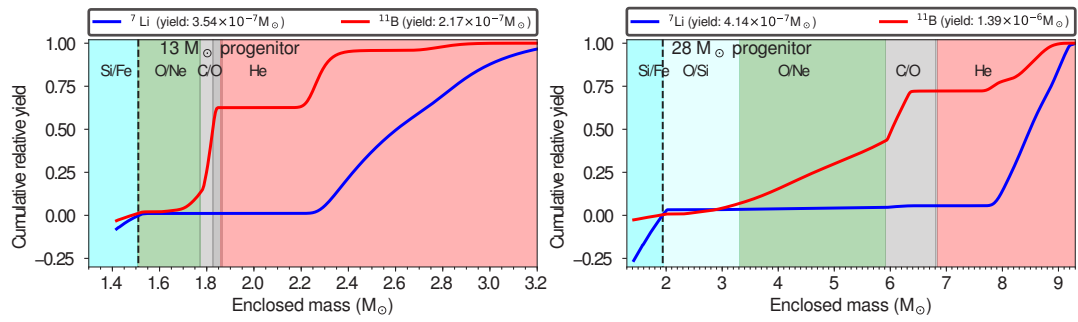

Figure 1. Cumulated relative yields for ${ }^{7} \mathrm{Li}$ and ${ }^{11} \mathrm{~B}$ over the profile of the 13 and $28 \mathrm{M}_{\odot}$ models considered here. The dashed black horizontal line indicates the position of the mass cut. Everything below the mass cut is commonly not counted as part of the ejecta and would not contribute to the yield. This part is shown here as negative.

mass cut, below which material is not counted for the ejecta. Figure 1 shows the cumulated yield normalized to the total yield taking into account a mass cut at the edge of the Fe core. It shows that ${ }^{7} \mathrm{Li}$ is almost exclusively made in the He shell while ${ }^{11} \mathrm{~B}$ has also important contributions from the $\mathrm{C}$ layer. The contribution from the $\alpha$ rich freeze out, which appears as negative when it is below the mass cut in Figure 1, is rather negligible in the $13 \mathrm{M}_{\odot}$ model but it increases for more massive progenitor stars. For the $28 \mathrm{M}_{\odot}$ progenitor an amount of more than $20 \%$ of the total yield of ${ }^{7} \mathrm{Li}$ could be contributed the innermost region that is hidden by the mass cut.

\section{The $v$ process in 2D}

The fate of the material from the innermost regions is sensitive to the details of the explosion dynamics. In two- and three dimensional simulations matter from this region below the mass cut can be ejected and convection could lead to a longer exposure of the material to the strong neutrino radiation close to the core. In order to explore the role of the $v$ process for the nucleosynthesis in the innermost ejecta, we have performed nuclear reaction network calculations for the thermodynamic histories of 4000 tracer particles from a self consistent 2D supernova simulation based on a $12 \mathrm{M}_{\odot}$ progenitor presented in ref. [12, 13]. The tracer particles are representative for the innermost $2 \mathrm{M}_{\odot}$ of the star, extending well into the lower part of the C-rich layer. Figure 2 shows the resulting mass fractions of ${ }^{7} \mathrm{Li}$ as a function of the initial position of the tracer particles and as projected onto the corresponding mass coordinate. The total yield is calculated by combining the results of the tracer particles for the inner region with the data from the $1 \mathrm{D}$ explosion model for the rest of the star. From these calculations we can see that material from the $\alpha$-rich freeze out, that gets enriched in ${ }^{7} \mathrm{Li}$ and ${ }^{11} \mathrm{~B}$ is ejected. However, for this progenitor the contribution to the total yield is negligible. Since we have seen in our 1D study that the production in the $\alpha$ rich freeze out can be more important for more massive progenitors, it is possible that a noticeable contribution from the innermost regions could result.

This work was partly supported by the Deutsche Forschungsgemeinschaft through contract SFB 1245, AH was supported by an Australian Research Council (ARC) Future Fellowship (FT120100363). WRH was supported by the U.S. Department of Energy, Office of Science, Offices of Nuclear Physics and the National Science Foundation Nuclear Theory Program (PHY-1516197). The Harris et al (2017) simulations were performed with resources of the National Energy Research Scientific Computing Center, supported by the U.S. DOE Office of Science under Contract No. DEAC02-05CH11231. 

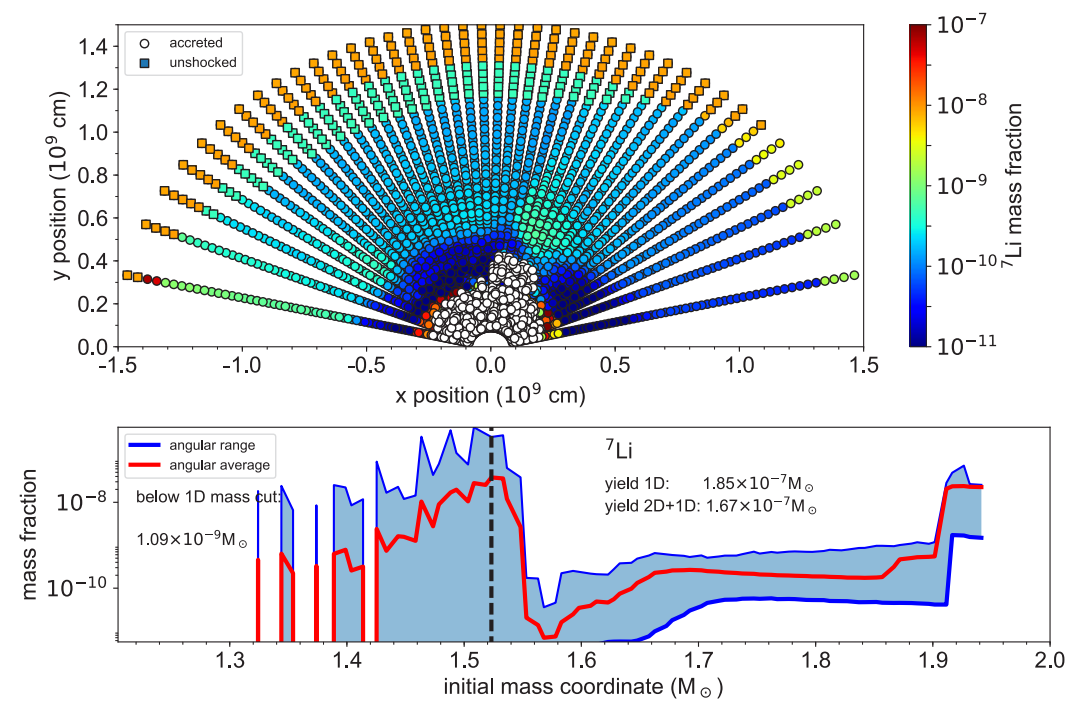

Figure 2. Mass fraction of ${ }^{7} \mathrm{Li}$ for the tracer particles from a self-consistent axisymmetric supernova simulation. The upper panel shows the mass fraction as color code for the initial position of the tracer particles. The lower panel shows the projection of these mass fractions onto the mass coordinate. Shown are is the range of mass fractions for different angles and the angular average.

\section{References}

[1] T. Rauscher, A. Heger, R.D. Hoffman, S.E. Woosley, Astrophys. J. 576, 323 (2002)

[2] S.E. Woosley, D.H. Hartmann, R.D. Hoffman, W.C. Haxton, Astrophys. J. 356, 272 (1990)

[3] A. Sieverding, L. Huther, G. Martínez-Pinedo, K. Langanke, A. Heger, Neutrino nucleosynthesis in core-collapse Supernova explosions, in European Physical Journal Web of Conferences (2016), Vol. 109 of European Physical Journal Web of Conferences, p. 06004

[4] A. Heger, E. Kolbe, W. Haxton, K. Langanke, G. Martínez-Pinedo, S.E. Woosley, Phys. Lett. B 606, 258 (2005)

[5] A. Mirizzi, I. Tamborra, H.T. Janka, N. Saviano, K. Scholberg, R. Bollig, L. Hüdepohl, S. Chakraborty, Riv. del Nuovo Cim. 39, 1 (2016)

[6] S.M. Austin, C. West, A. Heger, Phys. Rev. Lett. 112, 111101 (2014)

[7] K. Lodders, Astrophys. J. 591, 1220 (2003)

[8] P. Mohr, F. Käppeler, R. Gallino, Phys. Rev. C 75, 012802 (2007)

[9] T. Hayakawa, T. Kajino, S. Chiba, G.J. Mathews, Phys. Rev. C 81, 052801 (2010)

[10] T. Yoshida, T. Kajino, H. Yokomakura, K. Kimura, A. Takamura, D.H. Hartmann, Physical Review Letters 96, 091101 (2006)

[11] G.J. Mathews, T. Kajino, W. Aoki, W. Fujiya, J.B. Pitts, Phys. Rev. D 85, 105023 (2012)

[12] S.W. Bruenn, E.J. Lentz, W.R. Hix, A. Mezzacappa, J.A. Harris, O.E.B. Messer, E. Endeve, J.M. Blondin, M.A. Chertkow, E.J. Lingerfelt et al., ApJ 818, 123 (2016)

[13] J.A. Harris, W.R. Hix, M.A. Chertkow, C.T. Lee, E.J. Lentz, O.E.B. Messer, ApJ 843, 2 (2017) 